\title{
Universal finite-size effects in the two-dimensional asymmetric Coulomb gas on a sphere
}

\author{
L. Šamaj ${ }^{1,2}$ \\ Laboratoire de Physique Théorique, Université de Paris-Sud, \\ Bâtiment 210, 91405 Orsay Cedex, France
}

November 21, 2018

\begin{abstract}
We consider an asymmetric version of a two-dimensional Coulomb gas, made up of two species of pointlike particles with positive +1 and negative $-1 / Q(Q=$ $1,2, \ldots)$ charges; $Q=1$ corresponds to the symmetric two-component plasma and the limiting case $Q \rightarrow \infty$ is related to the one-component plasma. The system lives on the surface of a sphere, and it is studied in both canonical and grand-canonical ensembles. By combining the method of stereographic projection of the sphere onto an infinite plane with the technique of a renormalized Mayer series expansion it is explicitly shown that the finite-size expansions of the free energy and of the grand potential have the same universal term, independent of model's details. As a byproduct, the collapse temperature and the Kosterlitz-Thouless transition point (in the limit of a vanishing hard-core attached to particles) are conjectured for any value of $Q$.
\end{abstract}

LPT Orsay 01-26

${ }^{1}$ On leave from the Institute of Physics, Slovak Academy of Sciences, Bratislava, Slovakia

${ }^{2}$ E-mail addresses: Ladislav.Samaj@th.u-psud.fr and fyzimaes@savba.sk 


\section{Introduction}

The universal finite-size properties of two-dimensional (2D) critical systems with shortrange interactions among constituents are well understood within the principle of conformal invariance [1]-[5]. For a finite 2D system of characteristic size $R$ at critical point, the dimensionless grand potential $\beta \Omega[\beta=1 /(k T)$ is the inverse temperature $]$ has a large- $R$ expansion of the form

$$
\beta \Omega=A R^{2}+B R-\frac{c \chi}{6} \ln R+\ldots
$$

The coefficients $A$ and $B$ of the bulk and surface parts are non-universal. The coefficient of the $\ln R$-term is universal, dependent only on the conformal anomaly number $c$ of the critical theory and on the Euler number $\chi$ of the manifold (with a smooth metric and a smooth boundary) to which the system is confined. In general, $\chi=2-2 h-b$, where $h$ is the number of handles and $b$ the number of boundaries of the manifold $(\chi=2$ for a sphere, $\chi=1$ for a disk, $\chi=0$ for an annulus or a torus).

For lattice models and their continuum limits at critical point, the quantity (1.1) is usually called the dimensionless free energy, with the notation $\beta F$, but from a rigorous point of view those systems are usually treated in the grand-canonical ensemble (one sums over all spin configurations, particle occupations, etc.), with the grand potential as the generating functional. Here, we will consider a specific class of continuous fluid systems, which exhibit at any temperature the universal finite-size behaviour of type (1.1) in both grand-canonical (fixed fugacity, the grand potential) and canonical (fixed particle number

density, the Helmholtz free energy) ensembles. Although the two ensembles give the same thermodynamic behaviour in the thermodynamic limit, their finite-size corrections are expected to differ from one another. However, as will be documented in this work, at least for the sphere geometry of the confining manifold, the universal finite-size correction term is the same in both ensembles.

The fluids under consideration are the 2D Coulomb plasmas of charged particles, interacting through a long-range logarithmic potential. The one-component version (OCP) of equally, say unit, charged point particles in a uniform neutralizing background is formally related to the fractional quantum Hall effect [6]. The model is exactly solvable 
at the inverse temperature $\beta=2[7]$ by mapping onto free fermions. The symmetric two-component version (TCP) of oppositely \pm 1 charged pointlike particles undergoes the collapse of positive-negative pairs of charges at $\beta=2$, which corresponds to the exactly solvable free-fermion point of the equivalent Thirring model [8]-10]. If a small hard core is attached to the particles in order to prevent the collapse, the 2D TCP (or Coulomb gas) undergoes the famous Kosterlitz-Thouless (K-T) transition [11 from a high-temperature conducting phase to a low-temperature insulating phase at around $\beta=4$.

The long-range tail of the Coulomb potential induces screening. Due to the screening effect, the bulk charge-charge correlations are short-ranged, exponential or even Gaussian. On the other hand, the same screening effect causes that the induced electrical-field correlations are long-ranged [12], [13]. As a consequence, the free energy of the 2D OCP and the grand potential of the symmetric 2D TCP are supposed to exhibit, at any temperature of the conducting regime, a universal finite-size correction of type (1.1); this correction is opposite to the one occurring in the massless Gaussian field theory [5]) which has $c=1$. The arguments for a critical-like behaviour were first given for Coulomb gases with periodic boundary conditions [14], then for Coulomb gases confined to a domain by plain hard walls [15], by ideal-conductor boundaries [16], and finally by ideal-dielectric boundaries [17], [18]. In all cases, the explicit checks of the predicted finite-size universal behaviour were done at the exactly solvable inverse temperature $\beta=2$ for both OCP and TCP with various geometries of confining domains.

The general derivations of the universal finite-size correction term in refs. [14-[18] were based on plausible, but not rigorously justified, heuristic arguments. Very recently, a direct derivation was made for the specific case of Coulomb systems living on the surface of a sphere [where, since there is no boundary, $B=0$ in (1.1)]. By combining the method of stereographic projection of the sphere onto an infinite plane with linear response theory (TCP, ref. [19]) or with density functional approach (OCP, ref. [20]), the prefactor to the universal $\ln R$ correction term was related to the second moment of the short-range part of the density-density direct correlation function $c_{S R}$ for the planar system. This second moment, representing a new sum rule for the corresponding density correlation function, was evaluated explicitly for both OCP [21] and TCP [22 by using a renormalized Mayer 
expansion [23], [24], namely by observing a remarkable "cancelation property" of specific families of renormalized $c_{S R}$-diagrams. The obtained results confirm quantitatively the prediction of conformal invariance (1.1) for a critical system as we had $c=-1$.

In the present paper, we study a more general asymmetric 2D Coulomb gas made up of two species of pointlike particles with positive and negative charges of arbitrary strengths $q_{\sigma}(\sigma=1,2)$. Without any loss of generality, we can choose

$$
q_{1}=+1 \quad \text { and } \quad q_{2}=-1 / Q
$$

with $Q=1,2, \ldots$ a positive integer. The model interpolates between the symmetric TCP $(Q=1)$ and the OCP, obtained as the limiting $Q \rightarrow \infty$ case after subtracting the kinetic energy of the 2-species. For the asymmetric Coulomb gas confined to a sphere, by combining the method of stereographic projection [19], 20] with the technique of the renormalized Mayer expansion [21]-24] we prove that the prefactor to the universal $\ln R$ term in (1.1) does not depend on the parameter $Q$. The same universal term is obtained in both canonical (free energy) and grand-canonical (grand potential) ensembles, although in the previous studies of Coulomb systems the choice of the ensemble seemed to be relevant: the exact solution at $\beta=2$ and finite-size corrections were obtained strictly in the canonical ensemble for the OCP and in the grand-canonical ensemble for the TCP.

From a technical point of view, the asymmetry in the strength of particle charges induces an asymmetry in particle correlations: two independent two-particle combinations $11=22$ and $12=21$ in the symmetric TCP are replaced by the three ones 11, 22 and $12=21$. The method of stereographic projection [19], [20] is shown to imply a new "missing" interrelation among the second moments of species pair correlations. This, together with a generalization of the "cancelation" phenomena in the renormalized Mayer expansion, helps us to extend new sum rules for the plane OCP [21] and symmetric TCP 222 to the general asymmetric plasma and thus to find the prefactor to the universal finite-size correction for the latter model.

In their pioneering work about the asymmetric 2D Coulomb gas [25], the authors predicted a successive series of temperatures at which partial recombinations (or collapses) of positive-negative pairs of charges occur, with a consequent impact on the shortdistance behaviour of like-species correlations and on the equation of state (discontinuities 
of $\partial p / \partial \beta, p$ is the pressure, were suggested). However, the exact solutions of thermodynamics for the symmetric $Q=1$ [26] and the first asymmetric $Q=2$ [27] cases give only one singularity for the pressure at one collapse temperature, related to a short-distance instability of the configuration integral for a neutral cluster formed of one particle of species 1 and $Q$ particles of species 2 . The collapse temperature is explicitly derived for any $Q$ in this work. If one attach a hard-core $a$ to the particles, the system remains stable also below the collapse temperature, and can reach the K-T phase transition. In the limit of vanishing $a$, the presence of the K-T transition in the symmetric TCP is indicated by a divergence of the second moment of truncated density-density correlations [19]. Having at disposal the generalization of this second moment sum rule, we suggest the position of the $\mathrm{K}-\mathrm{T}$ transition temperature for any value of $Q$.

The paper is organized as follows. The method of stereographic projection in the grand-canonical [19] and canonical [20] ensembles is briefly generalized to the asymmetric 2D Coulomb gas in sections 2 and 3, respectively. Section 4 deals with the renormalized Mayer expansion. The final results, conjectures and some concluding remarks are given in section 5 .

\section{Grand-canonical ensemble}

On the surface of a sphere of radius $R$, two particles $i$ of species $\sigma_{i}$ and $j$ of species $\sigma_{j}$ interact via the Coulomb interaction $-q_{\sigma_{i}} q_{\sigma_{j}} \ln \left[(2 R / L) \sin \left(\theta_{i j} / 2\right)\right]$, where $L$ is a length constant set for simplicity to unity and $\theta_{i j}$ is the angular distance of particles [28]. The particle charges belong to the two-component set given by equation (1.2). Under the neutrality condition, the grand partition function reads

$$
\begin{aligned}
\Xi= & 1+z_{1}\left(z_{2}\right)^{Q} R^{2(1+Q)} \\
& \times \int \mathrm{d} \Omega_{0}^{(1)} \mathrm{d} \Omega_{1}^{(2)} \ldots \mathrm{d} \Omega_{Q}^{(2)} \frac{\prod_{(i<j)=1}^{Q}\left(2 R \sin \frac{\theta_{i j}}{2}\right)^{\beta / Q^{2}}}{\prod_{j=1}^{Q}\left(2 R \sin \frac{\theta_{0 j}}{2}\right)^{\beta / Q}}+\ldots,
\end{aligned}
$$

where $z_{\sigma}(\sigma=1,2)$ are the species fugacities and $\mathrm{d} \Omega_{i}^{(\sigma)}$ is an angle element around the position of particle $i$ of type $\sigma$. The term in (2.1) containing $N$ particles of type 1 and $Q N$ 
particles of type 2 equals $\left[z_{1} z_{2}^{Q} R^{2(1+Q)\left(1-\frac{\beta}{4 Q}\right)}\right]^{N}$ times a dimensionless integral dependent only on $\beta$ and $Q$. Thus, $\ln \Xi$ obeys the homogeneity relations

$$
z_{1} \frac{\partial}{\partial z_{1}} \ln \Xi=\frac{1}{Q} z_{2} \frac{\partial}{\partial z_{2}} \ln \Xi=\frac{1}{2(1+Q)}\left(1-\frac{\beta}{4 Q}\right)^{-1} R \frac{\partial \ln \Xi}{\partial R} .
$$

Let us denote by $N_{\sigma}^{(S)}$ the number of particles of type $\sigma$ on the sphere of radius $R$; $N_{\sigma}^{(S)}=4 \pi R^{2} \times$ the constant density $n_{\sigma}^{(S)}$. Since

$$
N_{\sigma}^{(S)}=z_{\sigma} \frac{\partial}{\partial z_{\sigma}} \ln \Xi, \quad \sigma=1,2
$$

one gets the relations

$$
\begin{aligned}
n_{1}^{(S)} & =\frac{1}{8 \pi(1+Q)}\left(1-\frac{\beta}{4 Q}\right)^{-1} \frac{1}{R} \frac{\partial}{\partial R} \ln \Xi \\
n_{2}^{(S)} & =\frac{Q}{8 \pi(1+Q)}\left(1-\frac{\beta}{4 Q}\right)^{-1} \frac{1}{R} \frac{\partial}{\partial R} \ln \Xi
\end{aligned}
$$

satisfying the electroneutrality condition $\sum_{\sigma} q_{\sigma} n_{\sigma}^{(S)}=0$.

For fixed fugacities $z_{\sigma}$ and in the large- $R$ limit, $\ln \Xi$ behaves like

$$
\ln \Xi=\beta p\left(4 \pi R^{2}\right)+f(R)+\ldots
$$

where $p$ is the bulk pressure of an infinite (plane) system and $f(R)$ is the leading finite-size correction. Substituting (2.5) into (2.4) one obtains, in the limit $R \rightarrow \infty$,

$$
\begin{aligned}
& n_{1}=\frac{1}{1+Q}\left(1-\frac{\beta}{4 Q}\right)^{-1} \beta p \\
& n_{2}=\frac{Q}{1+Q}\left(1-\frac{\beta}{4 Q}\right)^{-1} \beta p .
\end{aligned}
$$

where $n_{\sigma}$ is the number density of species $\sigma$ for the plane system. The equation of state follows immediately,

$$
\beta p=n\left(1-\frac{\beta}{4 Q}\right),
$$

where $n=\sum_{\sigma} n_{\sigma}$ is the total number density. The finite-size corrections to the species number densities then take the form

$$
\begin{aligned}
n_{1}^{(S)}-n_{1} & =\frac{1}{8 \pi(1+Q)}\left(1-\frac{\beta}{4 Q}\right)^{-1} \frac{1}{R} \frac{\partial}{\partial R} f(R), \\
n_{2}^{(S)}-n_{2} & =\frac{Q}{8 \pi(1+Q)}\left(1-\frac{\beta}{4 Q}\right)^{-1} \frac{1}{R} \frac{\partial}{\partial R} f(R) .
\end{aligned}
$$


Under stereographic projection of the sphere from its north pole onto an infinite plane tangent to its south pole $\boldsymbol{O}$, an area element $R^{2} \mathrm{~d} \Omega$ on the sphere is mapped onto $\mathrm{d}^{2} r$ on the plane according to

$$
R^{2} \mathrm{~d} \Omega=\frac{\mathrm{d}^{2} r}{\left(1+\frac{r^{2}}{4 R^{2}}\right)^{2}},
$$

and the angular distance $\theta_{i j}$ between two points on the sphere is expressible through the distance $\left|\boldsymbol{r}_{i}-\boldsymbol{r}_{j}\right|$ of their projections on the plane by

$$
2 R \sin \frac{\theta_{i j}}{2}=\frac{\left|\boldsymbol{r}_{i}-\boldsymbol{r}_{j}\right|}{\left(1+\frac{r_{i}^{2}}{4 R^{2}}\right)^{1 / 2}\left(1+\frac{r_{j}^{2}}{4 R^{2}}\right)^{1 / 2}} .
$$

After the stereographic transformation, (2.1) becomes the grand partition function of the underlying asymmetric Coulomb gas formulated on an infinite plane: besides the two-body interactions $-q_{\sigma_{i}} q_{\sigma_{j}} \ln \left|\boldsymbol{r}_{i}-\boldsymbol{r}_{j}\right|$, there is a species-dependent one-body potential acting on each particle,

$$
\begin{aligned}
& \beta V_{1}(\boldsymbol{r})=2\left(1-\frac{\beta}{4}\right) \ln \left(1+\frac{r^{2}}{4 R^{2}}\right) \underset{R \rightarrow \infty}{\simeq}\left(1-\frac{\beta}{4}\right) \frac{r^{2}}{2 R^{2}}, \\
& \beta V_{2}(\boldsymbol{r})=2\left(1-\frac{\beta}{4 Q^{2}}\right) \ln \left(1+\frac{r^{2}}{4 R^{2}}\right) \underset{R \rightarrow \infty}{\simeq}\left(1-\frac{\beta}{4 Q^{2}}\right) \frac{r^{2}}{2 R^{2}},
\end{aligned}
$$

The density shift of the planar system at the origin $\boldsymbol{O}$, due to the one-body potentials $V_{\sigma}(\boldsymbol{r})$, is given by linear response theory,

$$
\delta n_{\sigma}(\boldsymbol{O})=-\sum_{\sigma^{\prime}} \int \mathrm{d}^{2} r\left\langle\hat{n}_{\sigma}(\boldsymbol{O}) \hat{n}_{\sigma^{\prime}}(\boldsymbol{r})\right\rangle^{\mathrm{T}} \beta V_{\sigma^{\prime}}(\boldsymbol{r})
$$

where $\hat{n}_{\sigma}(\boldsymbol{r})=\sum_{i} \delta_{\sigma, \sigma_{i}} \delta\left(\boldsymbol{r}-\boldsymbol{r}_{i}\right)$ is the microscopic number density of species $\sigma$ at $\boldsymbol{r}$ and the truncated statistical average is taken in the unperturbed planar system. Let us introduce the notation

$$
I_{\sigma \sigma^{\prime}}=\int \mathrm{d}^{2} r r^{2}\left\langle\hat{n}_{\sigma}(\boldsymbol{O}) \hat{n}_{\sigma^{\prime}}(\boldsymbol{r})\right\rangle^{\mathrm{T}}
$$

for the second moment of the species-density correlation functions, with the obvious symmetry $I_{\sigma \sigma^{\prime}}=I_{\sigma^{\prime} \sigma}$. Since the density on the sphere equals to its projection on the plane at the south pole $\boldsymbol{O}$, it holds

$$
\delta n_{\sigma}(\boldsymbol{O})=n_{\sigma}^{(S)}-n_{\sigma}
$$


Inserting (2.11) into (2.12) and considering then (2.14) in (2.8), one finally gets

$$
\begin{aligned}
f(R)= & -C \ln R \\
C= & 2 \pi(1+Q)\left(1-\frac{\beta}{4 Q}\right) \\
& \times\left[\left(1-\frac{\beta}{4}\right)\left(I_{11}+\frac{1}{Q} I_{21}\right)+\left(1-\frac{\beta}{4 Q^{2}}\right)\left(I_{12}+\frac{1}{Q} I_{22}\right)\right] .
\end{aligned}
$$

Simultaneously, as a by-product, an important interrelation among I's results:

$$
\left(1-\frac{\beta}{4}\right)\left(I_{11}-\frac{1}{Q} I_{21}\right)=\left(1-\frac{\beta}{4 Q^{2}}\right)\left(\frac{1}{Q} I_{22}-I_{12}\right) \text {. }
$$

\section{Canonical ensemble}

In the canonical format, the stereographic projection maps the homogeneous particle densities $n_{\sigma}^{(S)}$ on the sphere onto the inhomogeneous ones on the plane,

$$
n_{\sigma}(\boldsymbol{r})=\frac{n_{\sigma}^{(S)}}{\left(1+\frac{r^{2}}{4 R^{2}}\right)^{2}} \underset{R \rightarrow \infty}{\simeq} n_{\sigma}^{(S)}\left(1-\frac{r^{2}}{2 R^{2}}\right) .
$$

The local chemical potential of particles $\sigma$ on the plane is given by

$$
\mu_{\sigma}(\boldsymbol{r})=\frac{\delta F[n]}{\delta n_{\sigma}(\boldsymbol{r})}
$$

where $F$ is the free energy functional. According to the elementary fluid theory [29],

$$
\begin{aligned}
\beta \frac{\delta \mu_{\sigma}(\boldsymbol{r})}{\delta n_{\sigma^{\prime}}\left(\boldsymbol{r}^{\prime}\right)} & =\frac{\delta^{2} \beta F[n]}{\delta n_{\sigma}(\boldsymbol{r}) \delta n_{\sigma^{\prime}}\left(\boldsymbol{r}^{\prime}\right)} \\
& =-c_{\sigma \sigma^{\prime}}\left(\boldsymbol{r}, \boldsymbol{r}^{\prime}\right)+\frac{\delta\left(\boldsymbol{r}-\boldsymbol{r}^{\prime}\right)}{n_{\sigma}(\boldsymbol{r})} \delta_{\sigma, \sigma^{\prime}}
\end{aligned}
$$

where $c$ stands for the direct correlation function. The chemical potentials of the asymmetric Coulomb system on the sphere coincide with the ones at the origin of the plane system, $\mu_{\sigma}(\boldsymbol{O})$, for the density profiles (3.1).

Let us start from a planar asymmetric Coulomb gas with particle densities $n_{\sigma}^{(S)}$ and switch on the one-body potentials $V_{\sigma}(\boldsymbol{r})$ 2.11). According to (3.1), the density change at point $\boldsymbol{r}$ caused by these one-body potentials is $\delta n_{\sigma}(\boldsymbol{r}) \simeq-n_{\sigma}^{(S)} r^{2} /\left(2 R^{2}\right)$. Using (3.3), the 
induced shift in the chemical potential at the origin is

$$
\begin{aligned}
\beta \delta \mu_{\sigma}(\boldsymbol{O}) & =\sum_{\sigma^{\prime}} \int \mathrm{d}^{2} r\left[-c_{\sigma \sigma^{\prime}}(\boldsymbol{r})+\frac{\delta(\boldsymbol{r})}{n_{\sigma}(\boldsymbol{O})} \delta_{\sigma, \sigma^{\prime}}\right] \delta n_{\sigma^{\prime}}(\boldsymbol{r}) \\
& \simeq \frac{1}{2 R^{2}} \sum_{\sigma^{\prime}} n_{\sigma^{\prime}}^{(S)} \int \mathrm{d}^{2} r c_{\sigma \sigma^{\prime}}(\boldsymbol{r}) r^{2}
\end{aligned}
$$

Consequently,

$$
\beta \sum_{\sigma} N_{\sigma}^{(S)} \delta \mu_{\sigma}(\boldsymbol{O}) \simeq 2 \pi \sum_{\sigma, \sigma^{\prime}} n_{\sigma}^{(S)} n_{\sigma^{\prime}}^{(S)} \int \mathrm{d}^{2} r c_{\sigma \sigma^{\prime}}(\boldsymbol{r}) r^{2}
$$

We will now omit the superscript $(S)$ in the notation and denote $N=\sum_{\sigma} N_{\sigma}$. The free energy $F$ has the large- $N$ behaviour

$$
\beta F=A N+g(N)+\text { const }+\ldots .
$$

Since $\mu_{\sigma}=\partial F / \partial N_{\sigma}$,

$$
\beta \delta \mu_{\sigma} \simeq \frac{\partial g(N)}{\partial N}
$$

Taking into account that $\delta \mu_{\sigma}=\delta \mu_{\sigma}(\boldsymbol{O})$, eq. (3.5) then implies

$$
\begin{aligned}
g(N) & =D \ln N \\
D & =2 \pi \sum_{\sigma, \sigma^{\prime}} n_{\sigma} n_{\sigma^{\prime}} \int \mathrm{d}^{2} r c_{\sigma \sigma^{\prime}}(\boldsymbol{r}) r^{2}
\end{aligned}
$$

where $n_{\sigma}$ are the particle densities of the asymmetric Coulomb gas formulated on an infinite plane.

\section{Renormalized Mayer expansion}

We first review briefly the renormalized Mayer expansion in density (for details, see refs. [21] - 24]) for a general fluid composed of distinct species of particles $\{\sigma\}$ with the corresponding densities $\{n(\boldsymbol{r}, \sigma)\}$. The particles $i$ and $j$ interact through the pair potential $v\left(\boldsymbol{r}_{i}, \sigma_{i} \mid \boldsymbol{r}_{j}, \sigma_{j}\right)$; vector position $\boldsymbol{r}_{i}$ will be sometimes represented simply by $i$. The renormalization technique is based on an expansion of each Mayer function in the inverse temperature $\beta$, and on a consequent series resummation of two-coordinated field circles; 
by coordination of a circle we mean the number of bonds meeting at this circle. The renormalized $K$-bonds are given by

$$
\begin{aligned}
& K-\beta v
\end{aligned}
$$

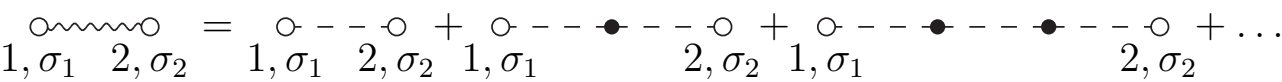

or, algebraically,

$$
\begin{aligned}
K\left(1, \sigma_{1} \mid 2, \sigma_{2}\right)= & {\left[-\beta v\left(1, \sigma_{1} \mid 2, \sigma_{2}\right)\right] } \\
& +\sum_{\sigma_{3}} \int\left[-\beta v\left(1, \sigma_{1} \mid 3, \sigma_{3}\right)\right] n\left(3, \sigma_{3}\right) K\left(3, \sigma_{3} \mid 2, \sigma_{2}\right) \mathrm{d} 3 .
\end{aligned}
$$

It is straightforward to verify by variation of (4.1) that it holds

$$
\frac{\delta K\left(1, \sigma_{1} \mid 2, \sigma_{2}\right)}{\delta n\left(3, \sigma_{3}\right)}=K\left(1, \sigma_{1} \mid 3, \sigma_{3}\right) K\left(3, \sigma_{3} \mid 2, \sigma_{2}\right) .
$$

The procedure of bond-renormalization transforms the ordinary Mayer representation of the excess Helmholtz free energy, defined by $-\beta F^{\mathrm{ex}}=-\beta F+\sum_{\sigma} \int \mathrm{d} 1[n(1, \sigma) \ln n(1, \sigma)-$ $n(1, \sigma)]$, into

$$
-\beta F^{\mathrm{ex}}=\bullet--\bullet+D^{(0)}[n]+\sum_{s=1}^{\infty} D^{(s)}[n],
$$

where

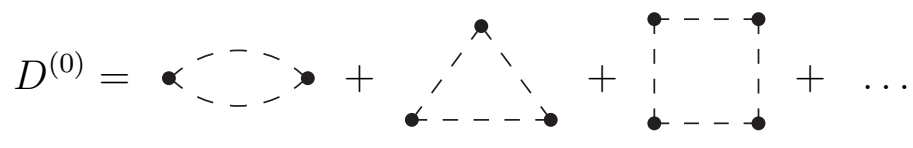

is the sum of all unrenormalized ring diagrams and

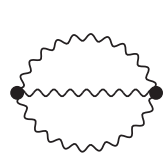

$D^{(1)}$

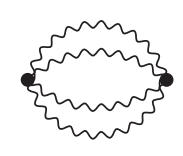

$D^{(2)}$

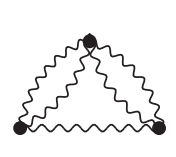

$D^{(3)}$

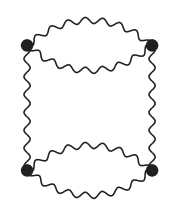

$D^{(4)}$

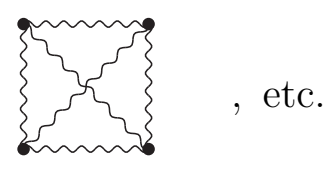

$D^{(5)}$

represent the set of all remaining completely renormalized graphs. The free energy is the generating functional for the direct correlation function $c$ in the sense that

$$
c\left(1, \sigma_{1} \mid 2, \sigma_{2}\right)=\frac{\delta^{2}\left(-\beta F^{\mathrm{ex}}\right)}{\delta n\left(1, \sigma_{1}\right) \delta n\left(2, \sigma_{2}\right)} .
$$

With regard to 4.3 ),

$$
c\left(1, \sigma_{1} \mid 2, \sigma_{2}\right)=-\beta v\left(1, \sigma_{1} \mid 2, \sigma_{2}\right)+\frac{1}{2 !} K^{2}\left(1, \sigma_{1} \mid 2, \sigma_{2}\right)+\sum_{s=1}^{\infty} c^{(s)}\left(1, \sigma_{1} \mid 2, \sigma_{2}\right),
$$


where $c^{(s)}\left(1, \sigma_{1} \mid 2, \sigma_{2}\right)$ represents the whole family of $\left(1, \sigma_{1}\right)\left(2, \sigma_{2}\right)$-rooted diagrams generated from a given $D^{(s)}$,

$$
c^{(s)}\left(1, \sigma_{1} \mid 2, \sigma_{2}\right)=\frac{\delta^{2} D^{(s)}}{\delta n\left(1, \sigma_{1}\right) \delta n\left(2, \sigma_{2}\right)} .
$$

To get explicitly $c^{(s)}$, one has to take into account that the functional derivative of $D^{(s)}$ with respect to the density field generates root circles not only at obvious field-circle positions, but also on $K$ bonds according to formula (4.2), causing their right $K-K$ division. The pair distribution function $h$, defined by

$$
n\left(1, \sigma_{1}\right) n\left(2, \sigma_{2}\right) h\left(1, \sigma_{1} \mid 2, \sigma_{2}\right)=\left\langle\hat{n}_{\sigma_{1}}(1) \hat{n}_{\sigma_{2}}(2)\right\rangle^{\mathrm{T}}-\delta_{\sigma_{1}, \sigma_{2}} \delta(1-2) n\left(1, \sigma_{1}\right)
$$

is related to $c$ via the Ornstein-Zernike $(\mathrm{OZ})$ equation

$$
\begin{aligned}
h\left(1, \sigma_{1} \mid 2, \sigma_{2}\right)= & c\left(1, \sigma_{1} \mid 2, \sigma_{2}\right) \\
& +\sum_{\sigma_{3}} \int \mathrm{d} 3 c\left(1, \sigma_{1} \mid 3, \sigma_{3}\right) n\left(3, \sigma_{3}\right) h\left(3, \sigma_{3} \mid 2, \sigma_{2}\right) .
\end{aligned}
$$

Let us return to the 2D asymmetric Coulomb gas with two kinds of particles $\sigma=1,2$ of charges (1.2), interacting via the logarithmic interaction

$$
\begin{aligned}
v\left(i, \sigma_{i} \mid j, \sigma_{j}\right) & =q_{\sigma_{i}} q_{\sigma_{j}} v(i, j), \\
v(i, j) & =-\ln |i-j| .
\end{aligned}
$$

We consider the infinite-volume limit, characterized by homogeneous densities $n(1, \sigma)=$ $n_{\sigma}$ constrained by the neutrality condition $\sum_{\sigma} q_{\sigma} n_{\sigma}=0$, so that

$$
n_{1}=n \frac{1}{1+Q}, \quad n_{2}=n \frac{Q}{1+Q}
$$

with $n$ being the total particle density. Two-body functions are both isotropic and translationally invariant, $c\left(1, \sigma_{1} \mid 2, \sigma_{2}\right)=c_{\sigma_{1} \sigma_{2}}(|1-2|), h\left(1, \sigma_{1} \mid 2, \sigma_{2}\right)=h_{\sigma_{1} \sigma_{2}}(|1-2|)$. From eq. (4.1) it follows that the renormalized bonds exhibit the same charge-dependence as the interaction under consideration (4.8),

$$
\begin{aligned}
K\left(1, \sigma_{1} \mid 2, \sigma_{2}\right) & =q_{\sigma_{1}} q_{\sigma_{2}} K(1,2), \\
K(1,2) & =-\beta K_{0}(\kappa|1-2|) .
\end{aligned}
$$


Here, $K_{0}$ is the modified Bessel function of second kind and $\kappa$ is the inverse Debye length defined by

$$
\kappa^{2}=2 \pi \beta \sum_{\sigma} q_{\sigma}^{2} n_{\sigma}
$$

The direct correlation function $c$, given by (4.5a), reads

$$
c_{\sigma_{1} \sigma_{2}}(|1-2|)=q_{\sigma_{1}} q_{\sigma_{2}} \beta \ln |1-2|+q_{\sigma_{1}}^{2} q_{\sigma_{2}}^{2} \frac{1}{2 !}\left[\beta K_{0}(\kappa|1-2|)\right]^{2}+\sum_{s=1}^{\infty} c_{\sigma_{1} \sigma_{2}}^{(s)}(|1-2|)
$$

For renormalized bonds of type (4.10), the $c^{(s)}$-families of diagrams exhibit, as separate units, remarkable "cancelation properties", regardless of the topology of the generating graph $D^{(s)}$. Let the given completely renormalized diagram $D^{(s)}(s=2,1, \ldots)$ be composed of $U$ skeleton vertices $i=1, \ldots, U$ of bond coordinations $\left\{\nu_{i}\right\}$ and $V=\sum_{i=1}^{U} \nu_{i} / 2$ bonds $a=1, \ldots, V$. Omitting topological factors, $D^{(s)}$ can be formally expressed as

$$
D^{(s)}[n]=\int \prod_{i=1}^{U}\left[\mathrm{~d} i \sum_{\sigma_{i}} n\left(i, \sigma_{i}\right)\right] \prod_{a=1}^{V} K\left(a_{1}, \sigma_{a_{1}} \mid a_{2}, \sigma_{a_{2}}\right),
$$

where $a_{1}, a_{2} \in\{1, \ldots, U\}, a_{1}<a_{2}$, denote the ordered pair of vertices joint by the $a$-bond. For the family $c^{(s)}$, generated from $D^{(s)}$ according to (4.5b), one gets

$$
\begin{array}{ll} 
& \sum_{\sigma, \sigma^{\prime}} n_{\sigma} n_{\sigma^{\prime}} c_{\sigma \sigma^{\prime}}^{(s)}\left(\left|\boldsymbol{r}-\boldsymbol{r}^{\prime}\right|\right)=\prod_{i=1}^{U}\left(\sum_{\sigma_{i}} q_{\sigma_{i}}^{\nu_{i}} n_{\sigma_{i}}\right) \\
(a) \quad \times \quad & {\left[\int \prod_{i} \mathrm{~d} i \sum_{\substack{i, j \\
(i \neq j)}} \delta(\boldsymbol{r}-i) \delta\left(\boldsymbol{r}^{\prime}-j\right) \prod_{a} K_{a}\right.} \\
(b) \quad & +\tilde{n} \int \prod_{i} \mathrm{~d} i \sum_{i} \delta(\boldsymbol{r}-i) \sum_{a} K\left(a_{1}, \boldsymbol{r}^{\prime}\right) K\left(\boldsymbol{r}^{\prime}, a_{2}\right) \prod_{b \neq a} K_{b} \\
(c) \quad & +\tilde{n} \int \prod_{i} \mathrm{~d} i \sum_{i} \delta\left(\boldsymbol{r}^{\prime}-i\right) \sum_{a} K\left(a_{1}, \boldsymbol{r}\right) K\left(\boldsymbol{r}, a_{2}\right) \prod_{b \neq a} K_{b} \\
(d) \quad & +(\tilde{n})^{2} \int \prod_{i} \mathrm{~d} i \sum_{a} K\left(a_{1}, \boldsymbol{r}^{\prime}\right) K\left(\boldsymbol{r}^{\prime}, \boldsymbol{r}\right) K\left(\boldsymbol{r}, a_{2}\right) \prod_{b \neq a} K_{b} \\
(e) \quad & +(\tilde{n})^{2} \int \prod_{i} \mathrm{~d} i \sum_{a} K\left(a_{1}, \boldsymbol{r}\right) K\left(\boldsymbol{r}, \boldsymbol{r}^{\prime}\right) K\left(\boldsymbol{r}^{\prime}, a_{2}\right) \prod_{b \neq a} K_{b} \\
(f) \quad &
\end{array}
$$

where we have applied the functional relation (4.2). Here, $K_{a} \equiv K\left(a_{1}, a_{2}\right)$ defined by (4.10a) and $\tilde{n}=\sum_{\sigma} q_{\sigma}^{2} n_{\sigma}=n / Q$. The (a) term in (4.14) corresponds to the creation of 
root points at two skeleton vertices, the next $(b, c)$ terms to one root circle generated at the skeleton and the other one at a bond, the $(d, e)$ terms to two root points at the same bond and the last $(f)$ term represents root points generated at two different renormalized bonds. Comparing (4.14) with formula (31) of ref. [21] and assuming that $\kappa^{2}=2 \pi \beta \tilde{n}$, it becomes clear that the rhs of (4.14) is proportional to $c^{(s)}\left(\left|\boldsymbol{r}-\boldsymbol{r}^{\prime}\right|\right)$ generated from $D^{(s)}$ for the case of the 2D OCP with mobile-particle density $\tilde{n}$. As was proven in ref. 21], the zeroth and second moments of such a $c^{(s)}$ vanish, regardless of the topology of the generating diagram $D^{(s)}$. Consequently, in our model,

$$
\begin{aligned}
\sum_{\sigma, \sigma^{\prime}} n_{\sigma} n_{\sigma^{\prime}} \int \mathrm{d}^{2} r c_{\sigma \sigma^{\prime}}^{(s)}(\boldsymbol{r}) & =0, \\
\sum_{\sigma, \sigma^{\prime}} n_{\sigma} n_{\sigma^{\prime}} \int \mathrm{d}^{2} r r^{2} c_{\sigma \sigma^{\prime}}^{(s)}(\boldsymbol{r}) & =0,
\end{aligned}
$$

for every $s=1,2, \ldots$

The 2D Fourier transform is defined by

$$
\begin{aligned}
& f(\boldsymbol{r})=\frac{1}{2 \pi} \int \mathrm{d}^{2} k \bar{f}(\boldsymbol{k}) \exp (\mathrm{i} \boldsymbol{k} \cdot \boldsymbol{r}), \\
& \bar{f}(\boldsymbol{k})=\frac{1}{2 \pi} \int \mathrm{d}^{2} r f(\boldsymbol{r}) \exp (-\mathrm{i} \boldsymbol{k} \cdot \boldsymbol{r}) .
\end{aligned}
$$

For a function with circular symmetry, whose Fourier component is analytic in $k$ around $k=0$, one has

$$
\begin{aligned}
\bar{f}(k) & =\sum_{j=0}^{\infty} \bar{f}^{(2 j)} k^{2 j} \\
\bar{f}^{(2 j)} & =\frac{(-1)^{j}}{4^{j}(j !)^{2}} \frac{1}{2 \pi} \int \mathrm{d}^{2} r r^{2 j} f(r) .
\end{aligned}
$$

The Fourier component of $K_{0}^{2}(r)$ is given by

$$
\begin{aligned}
\int \frac{\mathrm{d}^{2} r}{2 \pi} K_{0}^{2}(r) \exp (-i \boldsymbol{k} \cdot \boldsymbol{r}) & =\int_{0}^{\infty} \mathrm{d} r r J_{0}(k r) K_{0}^{2}(r) \\
& =\frac{\ln \left(k / 2+\sqrt{1+(k / 2)^{2}}\right)}{k \sqrt{1+(k / 2)^{2}}},
\end{aligned}
$$

with $J_{0}$ being the ordinary Bessel function. The Fourier transform of the direct correlation function $c$, eq. (4.12), thus reads

$$
\bar{c}_{\sigma \sigma^{\prime}}(k)=-q_{\sigma} q_{\sigma^{\prime}} \frac{\beta}{k^{2}}+\frac{\beta^{2}}{2 \kappa} q_{\sigma}^{2} q_{\sigma^{\prime}}^{2} \frac{\ln \left(k /(2 \kappa)+\sqrt{1+[k /(2 \kappa)]^{2}}\right)}{k \sqrt{1+[k /(2 \kappa)]^{2}}}+\sum_{s=1}^{\infty} \bar{c}_{\sigma \sigma^{\prime}}^{(s)}(k)
$$


where $\bar{c}_{\sigma \sigma^{\prime}}^{(s)}(k)$ are analytic functions of $k$. Regarding the neutrality condition, relations (4.15) can be reflected via

$$
\sum_{\sigma, \sigma^{\prime}} n_{\sigma} n_{\sigma^{\prime}} \bar{c}_{\sigma \sigma^{\prime}}(k)=\frac{\kappa^{2}}{16 \pi^{2}}-\frac{k^{2}}{96 \pi^{2}}+O\left(k^{4}\right)
$$

Note that the coefficient to the $k^{2}$-term is universal, i.e., independent of $\beta, n_{1}$ and $n_{2}$. In $\boldsymbol{k}$-space, the OZ equation (4.7) takes the form

$$
\bar{h}_{\sigma \sigma^{\prime}}(k)=\bar{c}_{\sigma \sigma^{\prime}}(k)+2 \pi \sum_{\sigma^{\prime \prime}} \bar{c}_{\sigma \sigma^{\prime \prime}}(k) n_{\sigma^{\prime \prime}} \bar{h}_{\sigma^{\prime \prime} \sigma^{\prime}}(k)
$$

The insertion of the leading term $\bar{c}_{\sigma \sigma^{\prime}}(k) \simeq-q_{\sigma} q_{\sigma^{\prime}} \beta / k^{2}$ into (4.21) leads to the zerothmoment (electroneutrality)

$$
\sum_{\sigma^{\prime}} q_{\sigma^{\prime}} n_{\sigma^{\prime}} \bar{h}_{\sigma^{\prime} \sigma}^{(0)}=-\frac{q_{\sigma}}{2 \pi}, \quad \sigma=1,2
$$

and the second-moment

$$
\sum_{\sigma, \sigma^{\prime}} q_{\sigma} n_{\sigma} q_{\sigma^{\prime}} n_{\sigma^{\prime}} \bar{h}_{\sigma \sigma^{\prime}}^{(2)}=\frac{1}{(2 \pi)^{2} \beta}
$$

Stillinger-Lovett sum rules [31], 32] for the truncated charge-charge correlation function $\langle\hat{\rho}(\boldsymbol{O}) \hat{\rho}(\boldsymbol{r})\rangle^{\mathrm{T}}$, where $\hat{\rho}(\boldsymbol{r})=\sum_{\sigma} q_{\sigma} \hat{n}_{\sigma}(\boldsymbol{r})$ is the total microscopic charge density at $\boldsymbol{r}$ [note the definition of $h$ in (4.6)].

To make use of (4.20), we introduce an auxilliary function

$$
\bar{f}_{\sigma}(k)=\sum_{\sigma^{\prime}} n_{\sigma^{\prime}} \bar{c}_{\sigma^{\prime} \sigma}(k)
$$

satisfying the relations

$$
\begin{aligned}
n_{1} \bar{f}_{1}^{(0)}+n_{2} \bar{f}_{2}^{(0)} & =\frac{\beta n}{8 \pi Q}, \\
n_{1} \bar{f}_{1}^{(2)}+n_{2} \bar{f}_{2}^{(2)} & =-\frac{1}{96 \pi^{2}} .
\end{aligned}
$$

At the lowest $k^{0}$ order, the $\mathrm{OZ}$ equation (4.21) gives

$$
\sum_{\sigma^{\prime}} n_{\sigma^{\prime}} \bar{h}_{\sigma^{\prime} \sigma}^{(0)}=\bar{f}_{\sigma}^{(0)}+2 \pi \sum_{\sigma^{\prime}} \bar{f}_{\sigma^{\prime}}^{(0)} n_{\sigma^{\prime}} \bar{h}_{\sigma^{\prime} \sigma}^{(0)}
$$


Combining (4.26) with (4.22), $\bar{f}_{1}^{(0)}$ and $\bar{f}_{2}^{(0)}$ occur only in the combination (4.25a), so they can be eliminated. Consequently, one gets explicitly

$$
\begin{aligned}
\bar{h}_{11}^{(0)} & =\frac{1}{2 \pi n[1-\beta /(4 Q)]}-\frac{1+Q}{2 \pi n}, \\
\bar{h}_{22}^{(0)} & =\frac{1}{2 \pi n[1-\beta /(4 Q)]}-\frac{1+Q}{2 \pi n Q}, \\
\bar{h}_{12}^{(0)} & =\frac{1}{2 \pi n[1-\beta /(4 Q)]} .
\end{aligned}
$$

Without going into details, these formulae imply the compressibility sum rule [25], [33], i.e., the zeroth moment of the truncated density-density correlation function $\langle\hat{n}(\boldsymbol{O}) \hat{n}(\boldsymbol{r})\rangle^{\mathrm{T}}$, where $\hat{n}(\boldsymbol{r})=\sum_{\sigma} \hat{n}_{\sigma}(\boldsymbol{r})$ is the total microscopic number density at $\boldsymbol{r}$.

At the next $k^{2}$ order, the OZ equation (4.21) gives

$$
\sum_{\sigma^{\prime}} n_{\sigma^{\prime}} \bar{h}_{\sigma^{\prime} \sigma}^{(2)}=\bar{f}_{\sigma}^{(2)}+2 \pi \sum_{\sigma^{\prime}} \bar{f}_{\sigma^{\prime}}^{(2)} n_{\sigma^{\prime}} \bar{h}_{\sigma^{\prime} \sigma}^{(0)}+2 \pi \sum_{\sigma^{\prime}} \bar{f}_{\sigma^{\prime}}^{(0)} n_{\sigma^{\prime}} \bar{h}_{\sigma^{\prime} \sigma}^{(2)}
$$

Inserting (4.27) into (4.28), $\bar{f}_{1}^{(2)}$ and $\bar{f}_{2}^{(2)}$ occur only in the combination (4.25b), so they can be eliminated. One obtains

$$
\begin{aligned}
\bar{h}_{11}^{(2)}+Q \bar{h}_{21}^{(2)} & =-\frac{1}{96 \pi^{2} n^{2}} \frac{1+Q}{1-\beta /(4 Q)}+2 \pi\left(\bar{f}_{1}^{(0)} \bar{h}_{11}^{(2)}+Q \bar{f}_{2}^{(0)} \bar{h}_{21}^{(2)}\right), \\
\bar{h}_{12}^{(2)}+Q \bar{h}_{22}^{(2)} & =-\frac{1}{96 \pi^{2} n^{2}} \frac{1+Q}{1-\beta /(4 Q)}+2 \pi\left(\bar{f}_{1}^{(0)} \bar{h}_{12}^{(2)}+Q \bar{f}_{2}^{(0)} \bar{h}_{22}^{(2)}\right) .
\end{aligned}
$$

From eqs. (4.6) and (4.17a) it follows that the second moments $I_{\sigma \sigma^{\prime}}$, defined by (2.13), are related to $\bar{h}_{\sigma \sigma^{\prime}}^{(2)}$ in the following way

$$
I_{\sigma \sigma^{\prime}}=-8 \pi n_{\sigma} n_{\sigma^{\prime}} \bar{h}_{\sigma \sigma^{\prime}}^{(2)}
$$

From the complete set of equations (4.29), 4.25a), (4.23) and the one (2.16) obtained by the stereographic projection, one finally finds that $\bar{f}_{1}^{(0)}=\beta /(8 \pi), \bar{f}_{2}^{(0)}=\beta /\left(8 \pi Q^{2}\right)$ and

$$
\begin{aligned}
& I_{11}=-\frac{2\left(3 \beta-8 Q^{2}\right)\left(\beta-6 Q^{2}\right)}{3 \beta \pi(\beta-4 Q)^{2}(1+Q)^{2}}, \\
& I_{22}=-\frac{2 Q^{4}(3 \beta-8)(\beta-6)}{3 \beta \pi(\beta-4 Q)^{2}(1+Q)^{2}}, \\
& I_{12}=\frac{2 Q^{2}\left[3 \beta^{2}-2 \beta\left(6-Q+6 Q^{2}\right)+48 Q^{2}\right]}{3 \beta \pi(\beta-4 Q)^{2}(1+Q)^{2}} .
\end{aligned}
$$


With regard to the definition of I's (2.13), the second moment of the number density correlation function is

$$
\int \mathrm{d}^{2} r r^{2}\langle\hat{n}(\boldsymbol{O}) \hat{n}(\boldsymbol{r})\rangle^{\mathrm{T}}=\frac{1-3 \beta(Q-1)^{2} /\left(2 Q^{2}\right)}{12 \pi[1-\beta /(4 Q)]^{2}}
$$

This is the generalization of the new sum rule derived for the symmetric $Q=1$ TCP in refs. [19] and [20].

\section{Final results and conclusion}

Inserting (4.31) into (2.15b), one gets $C=1 / 3$. With regard to (2.5) and (2.15a), the dimensionless grand potential, $\beta \Omega=-\ln \Xi$, has the large- $R$ expansion

$$
\beta \Omega=-\beta p\left(4 \pi R^{2}\right)+\frac{1}{3} \ln R+\text { const }+\ldots
$$

This expansion has the universal $\ln R$ term like in (1.1). Taking the sphere value of $\chi=2$, its prefactor corresponds to the conformal anomaly number $c=-1$ as was expected.

With regard to (4.17a), it follows from (4.20) that

$$
\sum_{\sigma, \sigma^{\prime}} n_{\sigma} n_{\sigma^{\prime}} \int \mathrm{d}^{2} r c_{\sigma \sigma^{\prime}}(r) r^{2}=\frac{1}{12 \pi} .
$$

Consequently, $D=1 / 6$ in (3.8), and (3.6) takes the form

$$
\beta F=A N+\frac{1}{6} \ln N+\text { const }+\ldots
$$

Since $N=4 \pi R^{2} n$, the dimensionless free energy $\beta F$ has the same universal correction term as $\beta \Omega$. Relations (5.1) and (5.3), valid for any asymmetric 2D Coulomb gas, are the main results of the present work.

As was promised in the Introduction, we now derive an expression for the collapse temperature as a function of $Q$. On the base of the exact results for the lowest cases $Q=1$ [26] and $Q=2$ [27], let us study the short-distance stability of the configuration integral of a neutral cluster formed of one particle of species 1, placed at the origin $\boldsymbol{O}$, and $Q$ particles of species 2:

$$
I_{\lambda}=\int_{\lambda}^{\Lambda} \prod_{i=1}^{Q} \mathrm{~d}^{2} r_{i} \frac{\prod_{(i<j)=1}^{Q}\left|\boldsymbol{r}_{i}-\boldsymbol{r}_{j}\right|^{\beta / Q^{2}}}{\prod_{i=1}^{Q}\left|\boldsymbol{r}_{i}\right|^{\beta / Q}}
$$


Here, $\Lambda$ is a screening length of the Coulomb system and $\lambda$ is a short-distance cutoff. After the change of variables $\boldsymbol{r}_{i}=\lambda \boldsymbol{r}_{i}^{\prime}, I_{\lambda}$ is expressible as follows

$$
I_{\lambda}=\lambda^{2 Q-\beta / 2-\beta /(2 Q)} \int_{1}^{\Lambda / \lambda} \prod_{i=1}^{Q} \mathrm{~d}^{2} r_{i}^{\prime} \frac{\prod_{(i<j)=1}^{Q}\left|\boldsymbol{r}_{i}^{\prime}-\boldsymbol{r}_{j}^{\prime}\right|^{\beta / Q^{2}}}{\prod_{i=1}^{Q}\left|\boldsymbol{r}_{i}^{\prime}\right|^{\beta / Q}}
$$

In the limit $\lambda \rightarrow 0, I_{\lambda}$ diverges as soon as $2 Q-\beta / 2-\beta /(2 Q)<0$, so that the asymmetric Coulomb gas is stable against collapse for $\beta<\beta_{\text {col }}$, with

$$
\beta_{\mathrm{col}}=\frac{4 Q^{2}}{1+Q}
$$

At the collapse point, although the density of particles (for a given fugacity) diverges, the truncated density correlation function $\langle\hat{n}(\boldsymbol{O}) \hat{n}(\boldsymbol{r})\rangle^{\mathrm{T}}$ is finite for $r \neq 0$. It was conjectured in ref. [3] and subsequently in ref. [19] that the formula for the second moment of $\langle\hat{n}(\boldsymbol{O}) \hat{n}(\boldsymbol{r})\rangle^{\mathrm{T}}$, having the dimension of (length $)^{0}$, might be analytically extended beyond the collapse point. This second moment remains finite up to the K-T phase transition (in the limit of vanishing hard-core): its divergence at the $\mathrm{K}$-T point is probably associated with a power-law decay of $\langle\hat{n}(\boldsymbol{O}) \hat{n}(\boldsymbol{r})\rangle^{\mathrm{T}}$ at asymptotically large distances. With regard to the sum rule (4.32), we therefore suggest that

$$
\beta_{\mathrm{KT}}=4 Q
$$

Note that $\beta_{\mathrm{col}}<\beta_{\mathrm{KT}}$, as it should be. Increasing $Q$, both $\beta_{\mathrm{col}}$ and $\beta_{\mathrm{KT}}$ tend to infinity, in full agreement with the fact that there exist neither the collapse phenomena nor the K-T phase transition for the one-component plasma.

In conclusion, we have studied an asymmetric two-dimensional Coulomb gas confined to the sphere. In both canonical and grand-canonical ensembles, the finite-size expansions of the free energy and of the grand potential are explicitly shown to possess the same universal term, independent of the model's details. The equivalence of both ensembles smears out the previously indicated difference in the treatment of finite-size corrections for the one-component and two-component plasmas. Whether such an explicit calculation of universal finite-size corrections is possible also for other geometries of confining domains is an open problem. 


\section{Acknowledgments}

I thank Bernard Jancovici for valuable discussions and careful reading of the manuscript. My stay in LPT Orsay is supported by a NATO fellowship. A partial support by Grant VEGA 2/7174/20 is acknowledged. 


\section{References}

[1] H.W.J. Blöte, J.L. Cardy, M.P. Nightingale, Phys. Rev. Lett. 56 (1986) 742.

[2] I. Affleck, Phys. Rev. Lett. 56 (1986) 746.

[3] J.L. Cardy, Phys. Rev. Lett. 60 (1988) 2709.

[4] J.L. Cardy, I. Peschel, Nucl. Phys. B 300 [FS22] (1988) 377.

[5] J.L. Cardy, in Fields, Strings and Critical Phenomena, Les Houches 1988, Editors E. Brézin and J. Zinn-Justin, North-Holland, Amsterdam, 1990.

[6] P.Di Francesco, M. Gaudin, C. Itzykson, F. Lesage, Int. J. Mod. Phys. A 9 (1994) 4257.

[7] B. Jancovici, Phys. Rev. Lett. 46 (1981) 386.

[8] M. Gaudin, J. Phys. (Paris) 46 (1985) 1027.

[9] F. Cornu, B. Jancovici, J. Stat. Phys. 49 (1987) 33.

[10] F. Cornu, B. Jancovici, J. Chem. Phys. 90 (1989) 2444.

[11] J.M. Kosterlitz, D.J. Thouless, J. Phys. C 6 (1973) 1181.

[12] J.L. Lebowitz, Ph.A. Martin, J. Stat. Phys. 34 (1984) 287.

[13] B. Jancovici, J. Stat. Phys. 80 (1995) 445.

[14] P.J. Forrester, J. Stat. Phys. 63 (1991) 491.

[15] B. Jancovici, G. Manificat, C. Pisani, J. Stat. Phys. 76 (1994) 307.

[16] B. Jancovici, G. Téllez, J. Stat. Phys. 82 (1996) 609.

[17] B. Jancovici, L. Šamaj, cond-mat/0101046, to appear in J. Stat. Phys. (July 2001).

[18] G. Téllez, cond-mat/0103122.

[19] B. Jancovici, J. Stat. Phys. 100 (2000) 201. 
[20] B. Jancovici, E. Trizac, Physica A 284 (2000) 241.

[21] P. Kalinay, P. Markoš, L. Šamaj, I. Travěnec, J. Stat. Phys. 98 (2000) 639.

[22] B. Jancovici, P. Kalinay, L. Šamaj, Physica A 279 (2000) 260.

[23] H.L. Friedman, Ionic Solution Theory, Interscience, New York, 1962.

[24] C. Deutsch, M. Lavaud, Phys. Rev. A 9 (1974) 2598.

[25] J.P. Hansen, P. Viot, J. Stat. Phys. 38 (1985) 823.

[26] L. Šamaj, I. Travěnec, J. Stat. Phys. 101 (2000) 713.

[27] L. Šamaj, in preparation.

[28] J.M. Caillol, J. Physique 42 (1981) L245.

[29] J.P. Hansen, I.R. McDonald, Theory of Simple Liquids, 2nd Edition, Academic Press, London, 1990.

[30] Ph.A. Martin, Rev. Mod. Phys. 60 (1988) 1075.

[31] F.H. Stillinger, R. Lovett, J. Chem. Phys. 48 (1968) 3858.

[32] F.H. Stillinger, R. Lovett, J. Chem. Phys. 49 (1968) 1991.

[33] P. Vieillefosse, J.P. Hansen, Phys. Rev. A 12 (1975) 1106. 